\section{Is there a lexicality component in the word superiority effect?}

\author{
LESLIE HENDERSON \\ The Hatfield Polytechnic \\ Hatfield AL10 9AB, England
}

Much attention has been lavished recently on word superiority effects in the hope that these phenomena can be made to yield information about the organization of skilled information processing in reading. In one important approach, an attempt is made to isolate and to specify those structural attributes of words that give rise to the advantage of words over random letter arrays that is found in various recognition tasks. A variety of positions have been taken on this question of the structural basis (or bases) of word superiority. We can usefully distinguish between those accounts that center upon lexicality, that is, the possession by a string of letters of an entry in a mental lexicon that is visually addressable, and those, on the other hand, that center upon the orthographic structuredness of a string. Orthographic factors can, in turn, be subdivided into visible structure, such as is represented by positional or sequential redundancy, and pronounceability. Adjudication between these positions is a matter of some theoretical importance, since it is reasonable to suppose that success in identifying the structural factors upon which word superiority depends would allow us to place strong constraints upon the possibilities in terms of underlying mechanisms.

Since the studies of tachistoscopic report by Gibson, Pick, Osser, and Hammond (1962) and of samedifferent judgments by Barron and Pittenger (1974), it has become clear that some aspect of orthographic structures can act as a sufficient cause of word superiority, since nonlexical pseudowords show a performance advantage over unstructured letter strings. The question of whether lexicality (real words vs. pseudowords) also exerts an effect on performance evidently remains in dispute.

A strong position on the lexicality question has recently been taken by Carr, Posner, Pollatsek, and Snyder (1979) on the basis of experiments using the same-different task. They assert that the efficiency of judgments based on visual codes is not influenced by lexicality ("familiarity," in their nomenclature). They argue: "It now appears that earlier findings of visual familiarity effects may be attributed to response biases resulting from the activation of higherlevel codes sensitive to familiarity, and to the use of

This research was supported by Grant HR 5482 from the Social Science Research Council (U.K.). small sets of training stimuli that allowed subjects to induce orthographic-like rules." Furthermore, they go on to suggest that the negative results that they obtain "reconcile an inconsistent literature." Finally, they draw some very strong and general implications from their experiments, asserting that their conclusions "may lead to changes in notions of how effective various kinds of visual training are likely to be at different stages in the acquisition of reading skill." (All quotations are taken from the summary of Carr et al., 1979.)

It is the purpose of this note to examine the conclusions drawn by Carr et al. from their visual matching task. First, I shall attempt to show that their tests of a lexical familiarity effect are inadequate. Their selection of stimuli renders the tests insensitive. Aspects of their design may have minimized the efficacy of a lexical strategy. Moreover, one of their manipulations, in which various amounts of experimental familiarization are provided for nonwords, has no necessary bearing on lexical familiarity. In a second section, I consider Carr et al.'s claim to have resolved apparent inconsistencies in the previous literature. In opposition to their conclusions, I show that the previous literature on same-different judgments, together with that on tachistoscopic thresholds, requires the conclusion that lexicality is a sufficient cause of perceptual facilitation. Many studies report lexicality effects that cannot be ascribed to response bias. The inconsistencies across studies as to whether an effect of lexicality is obtained can indeed be resolved. This is achieved by a systematic account of the conditions that need to be fulfilled for an effect to be obtained.

\section{Testing for an Effect of Lexicality}

In this section, I advance three criticisms of the adequacy of Carr et al.'s tests for a lexicality effect. These criticisms focus, in turn, on Carr et al.'s failure to control word frequency, the restriction of their test to three-letter stimuli, and their assumption that repetition within an experiment can be treated as equivalent to lexical frequency.

In a sense, the contrast between words and pseudowords is based on word frequency, insofar as the ideal pseudoword can be regarded as a word of zero frequency. The point of this way of expressing lexicality is that it helps us to see that any adequate test of a lexicality effect requires a maximization of word frequency. It is well known that within the population of real words, frequency effects are difficult to detect at low scale values. The finding that words of medium or low frequency behave like pseudowords is therefore of very little interest. Yet the word stimuli on which Carr et al. base their main test con- 
sist of 30 items, with a median frequency of only 13 occurrences/million, as assessed by the ThorndikeLorge (1944) G scale (these stimuli are listed in Table 1 of their paper). Furthermore, 5 of their 30 word stimuli have frequency values of less than 1 occurrence/ million. In the case of the items BOP and BAM, this would appear to be because they are not, in fact, lexical words. Moreover, there already exists evidence that the control of lexical frequency is critical, since Bruder (1978) has clearly shown that, in a matching experiment in which high-frequency words show an advantage over pseudowords, low-frequency words (less than 10/million) do not. Bruder's lowfrequency cut point would include $43 \%$ of the Carr et al. word stimuli.

Carr et al. employed four conditions so that a lexicality effect could be tested both by comparing words with pseudowords and by comparing meaningful, but unpronounceable, abbreviations with meaningless, unpronounceable strings. They arrived at their word stimuli by permutating the letters from their meaningful, but unpronounceable, stimuli so that FIB was derived from FBI. This latter class of stimuli is difficult to control for frequency, due to both the absence of systematic norms and the restrictions of the natural set from which such items must be drawn. Despite these difficulties, we require some reassurance that the abbreviations are sufficiently familiar to serve as the basis of a test of the lexicality hypothesis. Carr et al. offer no evidence on this point. However, inspection of their stimuli suggests that several of these supposedly familiar and meaningful stimuli can be discriminated from meaningless control stimuli only after considerable deliberation. These include IRS vs. SRI, GPA vs. APG, OSU vs. SUO, UFW vs. FWU, CPA vs. APC, and WPA vs. APW. Previous investigators using these sorts of stimuli have pretested them for familiarity (e.g., Henderson \& Chard, 1976), since, in the absence of reassurance that they are immediately identifiable, any failure to find an effect of the experimenterdefined lexicality manipulation is uninterpretable.

In short, the absence of any control of frequency and the fact that many of the words and almost all of the abbreviations used were of low frequency void the authors' claim to have carried out an adequate test of the lexicality hypothesis. ${ }^{1}$

As a minor additional point, it can be argued that the selection of stimuli is deficient in another respect. Consistent interactions, often substantial, have been shown between familiarity effects and string length in same-different judgments (Bruder, 1978; Chambers \& Forster, 1975; Eichelman, 1970; Henderson, 1974). These take the form of a diminution of the word superiority effect as letter length decreases. The three letter strings to which Carr et al. restrict their attention may therefore be an unfavorable condition for testing the effect of lexicality.

So far, we have seen that, by choosing short stim- ulus strings and by failing to ensure that the word strings were of high frequency, Carr et al. have accepted the null hypothesis for a lexicality effect on the basis of an insensitive test. This holds for their Experiments 1 and 2. In Experiment 3, they turn to a different manipulation, one in which massed practice with meaningless letter strings is used to induce a simulation of lexical familiarity. They show that this experimentally induced frequency effect has no influence on the efficiency with which strings are matched in the visual comparison task. This, they argue, represents further evidence against the hypothesis that "familiarity" affects the efficiency of visual matching.

What is odd about this experiment is the assumption that the property of lexicality, as embodied in the contrast of FIB with BIF or of FBI with IBF, can be treated as a factor equivalent to that arising from repeated experimental exposure to a nonword stimulus. Yet, Carr et al. discuss lexicality, and thus by implication word frequency, as if it could be simulated by manipulating the frequency of presentation of nonword stimuli in typing and matching tasks. Whether or not these two types of familiarity have equivalent effects and whether they are represented by the same structures in memory is a matter for investigation (see, for example, Scarborough, Cortese, \& Scarborough, 1977). However, it cannot be right to presume this equivalence in order to use the lack of effect of one factor as evidence of the ineffectiveness of the other.

Even in studies in which experimental repetition and word frequency are found to interact, as in Scarborough et al.'s (1977) work on lexical decision latencies, the evidence does not suggest that their effects are interchangeable. It is notable that in this work the full repetition effect is induced by a single prior trial. Furthermore, the repetition effect is larger for words than for nonwords.

Moreover, if repetition of nonwords had the effect of investing them with a familiarity that was equivalent to lexical status, then repeated trials with a small set of words and nonwords should abolish any effects of actual lexical status. Yet, Henderson's (1974) lexicality effect in visual matching survived several sessions of practice with a small, fixed set of stimuli.

\section{Previous Evidence of a Lexicality Effect}

Carr et al. claim to have effected a reconciliation of an inconsistent previous literature on lexicality effects. In what follows, I attempt to show that the weight of previous evidence implies the existence of a lexicality effect. Furthermore, the minority of anomalous findings in which a lexicality effect has not been obtained are generally those in which the test has been insensitive due to failure to control word frequency, as is the case in Carr et al.'s own study.

Carr et al. dismiss lexicality effects, where they occur, as the products of bias in criteria for respond- 
ing. Yet, it can be shown that in many previous demonstrations of a lexicality effect, criterion bias cannot be the cause. Such demonstrations can be found in studies of same-different judgments in which facilitatory effects in "same" judgments are not achieved at the cost of a deleterious effect in "different" judgments. They can also be found in studies of tachistoscopic report accuracy, to which Carr et al.'s criterion bias account does not apply. Moreover, large lexicality effects are most likely to be found when the lexicality factor is blocked, a manipulation that Carr et al. predict will diminish criterion bias as a source of a spurious lexicality effect.

Finally, I review the distinction between lexicality as a sufficient basis for the facilitation of performance and lexicality as a necessary basis. It is the former that serves as the appropriate test of the existence of a lexicality mechanism.

There exists a considerable literature that can be consulted as to the existence of lexicality effects. In Table 1, I present a summary of the evidence derived from nine previous papers in which a test of the lexicality effect is available. In six of these, the critical contrast is between words (e.g., FIB) and pseudowords (e.g., BIF). In the remaining three, the contrast is between meaningful compounds (e.g., FBI) and nonsense strings (e.g., IBF).

Considering first the latencies for "same" decisions, we find that lexical membership has a facilitatory effect in all of the 12 experiments listed. In only three of the studies does the effect fall below $50 \mathrm{msec}$. Of these, the only one to test this difference as a simple effect is the study by Barron and Henderson (1977). In their experiment, the effect was highly reliable.
Turning to the "different" decision latencies, we find less consistency. In all but one case, the lexicality effect is reduced, and in five cases, the lexicality effect is actually reversed.

Let us now consider this evidence in relation to the claim by Carr et al. that where a lexicality effect obtains, it is due simply to criterion bias, such that subjects are disposed toward responding "same" to any familiar stimuli. The expected consequence of such criterion bias is that an advantage won on "same" decisions should be lost on "different" decisions. Accordingly, a very minimal requirement of this hypothesis is that there should be a reversed familiarity effect for "different" decisions. Yet, this reversal obtains in only five of the experiments. Of these, we can confidently exclude criterion bias as a complete account of the lexicality effect in Chambers and Forster's (1975) Experiment 1, since the reversal for "different" decisions is trivial compared with the substantial positive effect on "same" decisions, and, indeed, the lexicality effect was reliable when the RTs were collapsed over type of response. In the remaining four experiments in which a crossover of the lexicality effect occurs, it is not possible to preclude an explanation exclusively based on criterion bias, although it is notable that in each case the facilitatory effect of lexicality on "same" decisions is greater than the inhibitory effect on "different" decisions.

Taking this considerable body of evidence as a whole, it is difficult to avoid the conclusion that a lexicality effect exists which cannot be dismissed as due to the criterion bias. This conclusion holds firmly for seven out of eight experiments in which words are compared with pseudowords. When abbrevia-

Table 1

Summary of Previous Findings in Investigations of the Lexicality Effect in Same-Different Judgments

\begin{tabular}{|c|c|c|c|c|c|}
\hline & \multirow[b]{2}{*}{$\begin{array}{l}\text { Experi- } \\
\text { ment }\end{array}$} & \multirow{2}{*}{$\begin{array}{l}\text { Number of } \\
\text { Letters in } \\
\text { the Stimuli }\end{array}$} & \multirow[b]{2}{*}{$\begin{array}{c}\text { Familiarity Blocked } \\
\text { or Randomized }\end{array}$} & \multicolumn{2}{|c|}{$\begin{array}{l}\text { Magnitude of the Facilitatory } \\
\text { Effect of Lexicality }\end{array}$} \\
\hline & & & & $\begin{array}{c}\text { Same } \\
\text { RT }\end{array}$ & $\begin{array}{c}\text { Different } \\
\text { RT } \\
\end{array}$ \\
\hline & \multicolumn{5}{|c|}{ Word vs. Pseudoword } \\
\hline Barron \& Pittenger (1974) & & 5 & Both & 55 & 12 \\
\hline Baron (1975) & 1 & 36 & Randomized & 23 & -10 \\
\hline Chambers \& Forster (1975) & 1 & $4-5$ & Randomized & 106 & -8 \\
\hline Chambers \& Forster (1975) & 2 & 5 & Randomized & 126 & 3 \\
\hline Taylor et al. (1977) & 2 & 6 & Randomized & 30 & 60 \\
\hline Taylor et al. (1977) & 3 & 5 & Blocked & 240 & 200 \\
\hline Bruder (1978) & 3 & 36 & Randomized & 172 & 58 \\
\hline \multirow[t]{2}{*}{ Barron \& Henderson (1977) } & 3 & 5 & Randomized & 42 & 19 \\
\hline & \multicolumn{5}{|c|}{ Abbreviations vs. Nonsense } \\
\hline Henderson (1974) & & $2-4$ & Blocked & 50 & 37 \\
\hline Henderson \& Chard (1976) & 1 & 3 & Randomized & 51 & -21 \\
\hline Henderson \& Chard (1976) & 2 & 3 & Randomized & 51 & -35 \\
\hline Seymour \& Jack (1978) & & 3 & Randomized & 60 & -45 \\
\hline
\end{tabular}

Note-These data are drawn only from conditions in which same-case stimuli were compared and in which the types of stimuli were equiprobable. Where word frequency was manipulated, only high-frequency words were considered. In the Barron and Henderson (1977) experiment, matching was on the basis of initial letter only. In the Taylor et al. (1977) study, the data were estimated from graphs of the original authors' results. Reaction times are given in milliseconds. 
tions are compared with nonsense strings, the question is more open to doubt. Clearly, however, there are further questions to be answered. The perceptual effect of lexicality would be more securely established if we could specify some of the systematic variables determining the considerable variations in the magnitude of the lexicality effect. Moreover, even though the lexicality effect cannot be wholly accounted for by criterion bias, it must be conceded that facilitation is invariably reduced and occasionally reversed for "different" decisions. Before turning to these questions, however, we must examine a further source of evidence against the criterion bias account of lexicality effects.

Carr et al. include the tachistoscopic threshold task in their discussion of familiarity effects, and there seem to be at least two reasons for including this paradigm in our review of the influence of lexical status. First, it has often been remarked that there is considerable parallelism between word superiority effects in these two paradigms (e.g., Henderson, 1977). Second, it is difficult to conceive how a criterion bias of the sort postulated by Carr et al. (1979) could enter into tachistoscopic report.

The literature on lexicality effects in tachistoscopic report is summarized in Table 2. Seven papers yield a total of 11 experiments in which words were compared with pseudowords. In seven of these, a reliable facilitatory effect of lexicality was obtained. The balance of evidence therefore favors the existence of a facilitatory effect of lexicality in a task in which criterion bias of the sort postulated by Carr et al. cannot occur.

Let us now consider whether some of the variations in the magnitude of the obtained lexicality effects can be accounted for by interactions with other systematic variables. There appear to be four candidates, namely, word frequency, blocking vs. randomizing of the lexicality factor, length of the letter string, and orthographic structuredness of the pseudowords. These are reviewed, in turn, below.

Table 2

Summary of Previous Findings in Investigations of Lexicality Effect (LE) in Tachistoscopic Recognition Tasks

\begin{tabular}{lcll}
\hline & $\begin{array}{c}\text { Experi- } \\
\text { ment }\end{array}$ & \\
\hline Baron \& Thurston (1973) & 1 & Randomized & No \\
Baron \& Thurston (1973) & 2 & Randomized & No \\
Manelis (1974) & 1 & Blocked & Yes \\
Manelis (1974) & 2 & Randomized & No \\
Manelis (1974) & 3 & Both & Yes \\
Spoehr \& Smith (1975) & 1 & Randomized & Yes \\
Spoehr \& Smith (1975) & 2 & Randomized & No \\
Juola, Leavitt, \& Choe (1974) & 1 & Randomized & Yes \\
McClelland (1976) & 1 & Blocked & Yes \\
McClelland \& Johnston (1977) & 1 & Blocked & Yes \\
Adams (1979) & 1 & Blocked & Yes \\
\hline
\end{tabular}

*Yes = reliable $;$ no $=$ not reliable .
We have already seen that the logic of the lexicality hypothesis requires that any test of it employ word stimuli in which lexical frequency is maximized. At an empirical level, the requirement is reinforced, since Bruder (1978) and Chambers and Forster (1975) have shown that lexical frequency determines the magnitude of the word advantage in the same-different task. Similarly, McClelland and Johnston (1977) found that the word advantage in tachistoscopic report held only for high-frequency words.

Turning now to studies in which a lexicality effect was not reliably obtained or in which the obtained effect might possibly be ascribed entirely to criterion bias, we find that these are generally studies in which word frequency was not controlled. We have already seen this to be true of Carr et al.'s stimuli. This deficiency obtains equally in the studies by Baron (1975) and Baron and Thurston (1973). These included "words" with very low frequencies, such as WRITHE, COON, and BOAS, and "nonwords" which in some cases turn out to have lexical status (VOLE, BRAD, PONS) and in other cases can be assigned a meaning (SORED, BONK, CONT, CONS). The remaining studies of same-different judgment in which there is some doubt about the existence of a truly perceptual effect of lexicality are those of Henderson and Chard (1976) and Seymour and Jack (1978). In these studies, control of lexical frequency was not possible, since they depended upon the FBI/IBF sort of contrast in which the meaningful items have to be drawn from a small natural set containing few highfrequency members. Again turning to the threshold task, we find that the negative finding of Spoehr and Smith (1975) was obtained in the absence of control of word frequency.

Blocking vs. randomizing appears to exert an equally clear influence on the detectability of the perceptual lexicality effect. Eight of the experiments listed in Tables 1 and 2 utilized blocking of the lexical status factor, and in none of these is the lexicality effect in doubt. It is noteworthy that, whereas Henderson and Chard (1976) and Seymour and Jack (1978) obtained weak evidence of the lexicality effect, with reversal for "different" decisions, Henderson (1974), using the same type of FBI/IBF contrast but with blocking of the lexical factor, found a clear lexicality effect for both response types. Moreover, Manelis (1974) specifically compared blocking and randomizing within a single study and found that, with randomization of the lexicality factor, its facilitatory effect was either reduced or eliminated. In a related manipulation, Taylor, Miller, and Juola (1977) varied the relative proportions of words and pseudowords within a randomized design using the samedifferent task. The lexicality effect for "same" decisions appears to be unaffected by this manipulation, but the lexical facilitation of "different" decisions 
increased with increases in the proportion of stimuli that were real words.

Carr et al.'s attempt to account for the vagaries of the lexicality effect consists of the assertion that lexicality effects may occur with randomized presentation but that, in such cases, they are attributable to criterion bias, whereas blocking may be expected to eliminate criterion bias and thus remove any sort of effect of lexicality. The present survey of the literature accords with the conclusion that blocked presentation reduces or eliminates criterion bias. However, from this point, our conclusions diverge sharply, since it is precisely the blocked condition that yields the most unambiguous evidence of a perceptual facilitation by lexicality (Henderson, 1974; Manelis, 1974; Taylor et al., 1977).

It is generally assumed that the effect of blocking is to allow the subject to preselect a strategy appropriate to the type of stimulus that is expected. There is, however, another possible way in which the sequencing of trials may also influence performance. It appears to be the case that many of the features of stimulus construction in these experiments conspire together to rob real words of their appearance of familiarity. Often the stimuli in experiments comparing performance on words with performance on nonwords share a common orthographic structure. (For example, $83 \%$ of the words employed by Carr et al., 1979, Experiments 1, 2, and 4, had CVC structure.) Furthermore, the pseudowords are commonly generated by changing a single vowel in the words. A consequence of this is that the experimental set of stimuli repeatedly sample a very restricted zone in orthographic (visual similarity) space. When a restricted zone of orthographic space is repeatedly sampled, this may result in a level of continuous activation of the members of that zone. Such a spread of activation is frequently postulated for semantic space, and at least one recent theory extends the notion to orthographic space (Glushko, 1979). Now, a consequence of this continuous activation may be satiation, by analogy with semantic satiation due to repeated presentation of a particular word, and this attenuation of lexical familiarity may be greater when the similarly structured stimuli consist of a random mixture of words and pseudowords. This effect is simulated in Table 3, from which the reader may get some experience of the induced attenuation of lexical familiarity by reading through the list of structurally similar words and pseudowords.

Table 3

Illustration of the Consequences for Perceived Lexical Status of Randomizing a Set of Word and Pseudoword Stimuli

\begin{tabular}{lllllllll} 
COD & CON & COR & COG & COM & COL & COT & COS & COP \\
CAG & CAD & CAR & CAN & CAM & CAL & CAP & CAT & CAS \\
CUL & CUM & CUN & CUG & CUD & CUR & CUT & CUS & CUP \\
\hline
\end{tabular}

Another factor that appears to interact with the word superiority effect in same-different judgments is the number of letters in the stimuli (Bruder, 1978; Chambers \& Forster, 1975; Eichelman, 1970; Henderson, 1974). It is noteworthy that those studies in which an overall facilitation by lexicality is in doubt are also those that use short stimulus strings. Thus, Henderson (1974) found no effect of lexicality for two-letter abbreviations, and the studies by Carr et al. (1979), Henderson and Chard (1976), and Seymour and Jack (1978) used only three-letter stimuli. Baron (1975) used stimuli of three, four, and five letters but did not analyze letter length.

So far, we have concentrated on factors that can explain the insensitivity of some studies to a lexicality effect, but, to balance matters, it should be pointed out that in the study by Taylor et al. (1977, Experiment 3 ), in which the lexicality effect was extraordinarily large, the pseudowords may have been less orthographically "legal" than the words (e.g., TAGRN and SNEPD). Nevertheless, this problem is by no means a general one, since most of the studies used simple rules such as vowel substitution to transform words into pseudowords, thereby controlling for orthographic structuredness.

\section{Conclusions}

Carr et al. (1979) report an absence of a lexicality effect, except when it can be dismissed as due to criterion bias. They argue that these negative conclusions are consistent with the previous literature. In the foregoing analysis, I have argued that the literature on same-different judgments, as well as the literature on tachistoscopic report accuracy, supports the alternative conclusion that lexical status exerts a perceptual facilitatory effect in these tasks. Furthermore, a detailed investigation of the previous literature reveals that the magnitude of this facilitatory effect depends on three other systematic variablesword frequency, blocking vs. randomizing of the lexicality factor, and number of letters in the stimulus. Armed with this realization, we can see why Carr et ai. were unable to detect evidence of the operation of a processing mechanism that makes use of lexicality, since their study employed values of all three variables that would be expected to minimize the lexicality effect.

Moreover, in two ancillary arguments, I have speculated that Carr et al.'s method of generating stimuli so that they occupy a small zone in orthographic space may lead to a satiation effect in which lexical familiarity is attenuated, and I have rejected repeated presentation of nonwords as a technique for inducing lexical familiarity.

There are several general implications that follow from this analysis. Of these, the nature of the lexicality mechanism lies outside the scope of this note 
(but see, for example, Henderson \& Chard, 1980; McClelland \& Johnston, 1977; Seymour \& Jack, 1978). Also, I shall not attempt an account of the vagaries of the effect of lexicality on "different" decisions. However, one point that can be briefly made here is that the currently prevalent fascination with the word superiority effect has tended to restrict consideration of such factors as lexicality to their potential role as agents of word superiority. However, it is likely that the word superiority effect can be sustained by a number of factors, lexical and orthographic. The word advantage is of interest in itself only insofar as it can provide existence proofs for mechanisms that utilize these various kinds of structure. Accordingly, the question of interest is whether a given factor serves as a sufficient cause, rather than a necessary cause, of word superiority. (It is for this reason that the FBI/IBF type of comparison, in which orthographic structure is absent as a factor, is so theoretically interesting, despite the practical difficulties of controlling word frequency, etc.) The moral in this is that if a recognition advantage is being used as a test of the existence of a lexicality mechanism, then the investigation should be designed so as to maximize the likelihood of any effect. Conversely, the absence of a lexicality effect has no certain bearing on the availability, in principle, of a lexical strategy. It merely establishes that, in the given circumstances, the performance was obtained by other strategies.

\section{REFERENCES}

Adams, M. J. Models of word recognition. Cognitive Psychology, 1979, 11, 133-176.

Baron, J. Successive stages in word iecognition. In P. M. A. Rabbitt \& S. Dornic (Eds.), Attention and performance $V$. London: Academic Press, 1975.

Baron, J., \& Thurston, I. An analysis of the word superiority effect. Cognitive Psychology, 1973, 4, 207-228.

Barron, R. W., \& Henderson, L. The effects of lexical and semantic information on same-different visual comparison of words. Memory \& Cognition, 1977, 5, 566-579.

Barron, R. W., \& Pittenger, J. B. The effect of orthographic structure and lexical meaning on same-different judgements. Quarterly Journal of Experimental Psychology, 1974, 26, 566-581.

BrUDER, G. A. Role of visual familiarity in the word-superiority effects obtained with the simultaneous-matching task. Journal of Experimental Psychology: Human Perception and Performance, 1978, 4, 88-100.

Carr, T. H., Posner, M. I., Pollatsek, A., \& Snyder, C. R. R. Orthography and familiarity effects in word processing. Journal of Experimental Psychology: General, 1979, 108, 389-414.

Chambers, S. M., \& Forster, K. I. Evidence for lexical access in a simultaneous matching task. Memory \& Cognition, 1975. 3, 549-559.

Eichelman, W. H. Familiarity effects in a simultaneous matching task. Journal of Experimental Psychology, 1970, 86, 275-282.

Gibson, E. J., Pick, A. D., Osser, H., \& Hammond, M. The role of grapheme-phoneme correspondence in the percep- tion of words. American Journal of Psychology, 1962, 75, 554-570.

Glushko, R. J. The organization and activation of orthographic knowledge in reading aloud. Journal of Experimental Psychology: Human Perception and Performance, 1979, 5, 674-691.

Henderson, L. A word superiority effect without orthographic assistance. Quarterly Journal of Experimental Psychology, 1974, 26, 30i-311.

Henderson, L. Word recognition. In N. S. Sutherland (Ed.), Tutorial essays in psychology (Vol. 1). Hillsdale, N.J: Erlbaum, 1977.

Henderson, L. \& Crard, M. J. On the nature of the facilitation of visual comparisons by lexical membership. Bulletin of the Psychonomic Society, 1976, 7, 432-434.

Henderson, L., \& Chard, M. J. The readere's implicit knowledge of orthographic structure. In U. Frith (Ed.), Cognitive processes in spelling. London: Academic Press, 1980.

Juola, J. F., Leavitt, D. D., \& Choe, C. S. Letter identification in word, nonword, and single-letter displays. Bulletin of the Psychonomic Society, 1974, 4, 278-280.

Manelis, L. The effect of meaningfulness in tachistoscopic word perception. Perception \& Psychophysics, 1974, 16, 182-192.

McClelland, J. L. Preliminary letter identification in the perception of words and nonwords. Journal of Experimental Psychology: Human Perception and Performance, 1976, 2, 80-91.

McClelland, J. L., \& Johnston, J. C. The role of familiar units in perception of words and nonwords. Perception \& $P_{S y}$ chophysics, 1977, 22, 249-261

Novik, N. Parallel processing in a word-nonword classification task. Journal of Experimental Psychology, 1974, 102, 1015-1020.

Scarborough, D. L., Cortese, C., \& Scarborough, H. S. Frequency and repetition effects in lexical memory. Journal of Experimental Psychology: Human Perception and Performance, $1977,3,1-17$.

SEymour, P. H. K., \& JACK, M. V. Effects of visual familiarity on "same" and "different" decision processes, Quarterly Journal of Experimental Psychology, 1978, 30, 455-470.

Spozhr, K. T., \& Smith, E. E. The role of orthographic and phonotactic rules in perceiving letter patterns. Journal of Experimental Psychology: Human Perception and Performance, 1975, 1, 21-24.

Taylor, G. A., Miller, T. J., \& JuolA, J. F. Isolating visual units in the perception of words and nonwords. Perception \& Psychophysic., 1977, 21, 377-386.

Thonndike, F. L., \& Lorge, I. The teacher's word book of 30,000 words. New York: Bureau of Publications, Teachers College, Columbia University, 1944.

\section{NOTE}

1. The fact that Carr et al. were able to obtain reliable differences between their words and pseudowords in other tasks calling for lexical or semantic judgments does not, of course, establish that the contrast between these stimuli was adequate to test for a lexicality effect in the visual matching task. Indeed, the extraordinary high error rates obtained in these tasks reinforce the argument that the lexicality contrast was not salient. For instance, in the lexical decision task, word-affirmative responses led to a $24 \%$ error rate. Furthermore, two sorts of evidence suggest that the word-affirmative decisions required extended lexical search. The speed advantage for word affirmation compared with pseudoword rejection is anomalously slight, and there is a failure to replicate Novik's (1974) finding of slower rejection of unpronounceable stimuli when they are meaningful abbreviations.

(Received for publication March 4, 1980; accepted April 8, 1980.) 